\title{
Effectiveness of Training in Changing Allied Health Students' Cultural Competency Skills
}

\author{
Gisela Van Kessel \\ University of South Australia, gisela.vankessel@unisa.edu.au \\ Caroline Fryer \\ University of South Australia, caroline.fryer@unisa.edu.au \\ Bernadette Flynn \\ University of South Australia, Bernadette.Flynn@unisa.edu.au \\ Katia Ferrar \\ La Trobe University, K.Ferrar@latrobe.edu.au \\ Susan Roush \\ University of Rhode Island, roush@uri.edu
}

Follow this and additional works at: https://nsuworks.nova.edu/ijahsp

Part of the Occupational Therapy Commons, Pharmacy and Pharmaceutical Sciences Commons, and the Physiotherapy Commons

\section{Recommended Citation}

Van Kessel G, Fryer C, Flynn B, Ferrar K, Roush S. Effectiveness of Training in Changing Allied Health Students' Cultural Competency Skills. The Internet Journal of Allied Health Sciences and Practice. 2022 Jan 03;20(1), Article 2.

This Literature Review is brought to you for free and open access by the College of Health Care Sciences at NSUWorks. It has been accepted for inclusion in Internet Journal of Allied Health Sciences and Practice by an authorized editor of NSUWorks. For more information, please contact nsuworks@nova.edu. 


\title{
Effectiveness of Training in Changing Allied Health Students' Cultural Competency Skills
}

\begin{abstract}
Purpose: Allied health students require cultural competence training to help them act as caring, ethical, and socially responsible health professionals. There is a lack of evidence for the effectiveness of cultural competence training changing the cultural skill level of allied health students or their behaviour in clinical practice. Method: We conducted a systematic search of the literature to examine the effectiveness of cultural competence training in changing allied health student cultural competency skills. CINAHL, Embase, ERIC, Medline and Scopus databases were searched from inception until August 2017. Search terms represented the population (e.g. allied health students), and cultural competence training. Two reviewers independently screened all retrieved studies and abstracts, then full text papers against inclusion criteria. All included studies were critically appraised, and data was extracted for analysis. Results: Of the initial 9,076 database hits, 15 intervention studies met the inclusion criteria; indicating this is a nascent area of research. Fourteen of the 15 studies demonstrated that cultural competence training provided to allied health students could significantly increase their perceived cultural competence skills, with effect sizes ranging from small to very large. Training was predominately conducted using traditional classroom teaching activities. The review identified that this field of research is dominated by the model and survey tool devised by Campinha-Bacote with a focus on skills required for patient assessment, rather than patient outcomes. Conclusions and possibly recommendations: Cultural competence training can improve allied health students' perceived level of cultural competence skills for clinical practice. To ensure an effective translation of skill development to clinical practice, tertiary institutions seeking to develop and evaluate cultural competency training programs should focus on teaching cultural competency skills that lead to improved patient outcomes.
\end{abstract}

\section{Author Bio(s)}

Dr Gisela van Kessel is an Associate Dean with University Online and was previously the program director for Physiotherapy in the Allied Health and Human Performance Unit, University of South Australia, AUS. Gisela is a registered physiotherapist in Australia.

Dr. Caroline Fryer is a senior lecturer with the Allied Health and Human Performance Unit at the University of South Australia, AUS. Caroline is a registered physiotherapist in Australia.

Ms. Bernadette Flynn was a Clinical Educator with the School of Health Sciences, University of South Australia, AUS. Bernie is a retired registered physiotherapist.

Dr Katia Ferrara is a Research Fellow with La Trobe University and The Australian Ballet and is also an Adjunct Senior Lecturer with the University of South Australia, AUS. Katia is a registered physiotherapist in Australia.

Dr Susan Roush was with the Department of Physical Therapy, University of Rhode Island, Kingston, USA and is now retired 


\title{
1IJAHSP \\ The Internet Journal of Allied Health Sciences and Practice \\ Dedicated to allied health professional practice and education
}

Vol. 19 No. 4 ISSN 1540-580X

\section{A Review of the Effectiveness of Training in Changing Allied Health Student Clinical Practice Cultural Competence Skills}

\author{
Gisela Van Kessel ${ }^{1}$ \\ Caroline Fryer ${ }^{1}$ \\ Bernadette Flynn ${ }^{1}$ \\ Katia Ferrar2 \\ Susan Roush ${ }^{3}$
}

1. University of South Australia

2. LaTrobe University

3. University of Rhode Island

Australia and United States

\begin{abstract}
Purpose: Allied health students require cultural competence training to help them act as caring, ethical, and socially responsible health professionals. There is a lack of evidence for the effectiveness of cultural competence training changing the cultural skill level of allied health students or their behaviour in clinical practice. Method: We conducted a systematic search of the literature to examine the effectiveness of cultural competence training in changing allied health student cultural competence skills. CINAHL, Embase, ERIC, Medline and Scopus databases were searched from inception until August 2017. Search terms represented the population (e.g. allied health students), and cultural competence training. Two reviewers independently screened all retrieved studies and abstracts, then full text studies against inclusion criteria. All included studies were critically appraised, and data was extracted for analysis. Results: Of the initial 9,076 database hits, 15 intervention studies met the inclusion criteria; indicating this is a nascent area of research. Fourteen of the 15 studies demonstrated that cultural competence training provided to allied health students could significantly increase their perceived cultural competence skills, with effect sizes ranging from small to very large. Training was predominately conducted using traditional classroom teaching activities. The review identified that this field of research is dominated by the model and survey tool devised by Campinha-Bacote with a focus on skills required for patient assessment, rather than patient outcomes. Conclusions and possibly recommendations: Cultural competence training can improve allied health students' perceived level of cultural competence skills for clinical practice. To ensure an effective translation of skill development to clinical practice, tertiary institutions seeking to develop and evaluate cultural competence training programs should focus on teaching cultural competence skills that lead to improved patient outcomes.
\end{abstract}

Key words: review, meta-analysis, cultural competence, allied health students, skills 


\section{INTRODUCTION}

The importance of providing culturally competent care is recognized by the professional accreditation bodies of most allied health profession disciplines. Practitioners are required to demonstrate their ability to service diverse patient populations and this requirement is reflected in the standards for professional training. Cultural competence has been defined as "the ability to work and communicate effectively and appropriately with people from culturally different backgrounds."1(p. 120) Cultural competence definitions within the allied health literature sometimes include the need to take a broader systems and policy approach to crosscultural work however, are most commonly defined as a set of internal attributes within the practitioner. ${ }^{2,3}$ These attributes include cultural attitudes, awareness, knowledge and skills. ${ }^{1}$ Cultural awareness is an individual's awareness of their own views; cultural knowledge encompasses the acquisition of knowledge of other cultures; and cultural skills are the behaviours required to interact effectively with people of other cultures. ${ }^{1}$

There are more than 18 cultural competence models presented within the health care literature, with the majority of these developed by the nursing profession using a literature review methodology. ${ }^{1}$ These models define cultural competence skills in health care in a variety of ways, including the ability to collect relevant cultural data regarding the client's presenting problem as well as accurately conduct a culturally-based physical assessment using communication skills. ${ }^{1}$

Previous reviews into effective cultural competence training have focused on the health care system or the competence of health professional staff, although some have included a few studies with students.,4,5,7 These reviews provide preliminary evidence that training can achieve a change in cultural competence awareness and knowledge. There is a lack of evidence for the effectiveness of cultural competence training changing the cultural skill level of students or their behaviour in clinical practice. In addition, the literature is weighted towards the experience of medical and nursing professions, so little is known about the effectiveness of training for allied health students. This study reports a review of the literature on the effectiveness of cultural competence training in changing allied health student clinical practice skills. We also sought to critique the research methods and summarise the training approaches being used in order to inform future curriculum design and research.

\section{METHOD}

This systematic review was prospectively registered with PROSPERO and is reported in accordance with the Preferred Reporting Items for Systematic Reviews and Meta Analyses Guidelines (PRISMA). ${ }^{8}$

Selection of search terms and databases was guided by an academic librarian. To capture both educational and heath literature, the CINAHL, Embase, ERIC, Medline and Scopus databases were searched from inception until August 2017. Search terms (including MESH headings when appropriate and truncations) were selected to represent the key research question themes of population (e.g. allied health students), and cultural competence training (see Appendix A). Culturally competent skills were defined as culturally responsive communication and behaviours used throughout clinical practice. Allied health students were defined as enrolled in a university health sciences course (not medical, dental or nursing) at bachelor's degree/Australian Qualifications Framework (AQF) Level 7 or higher and accredited by the relevant national accreditation body.

Inclusion criteria included: (a) the participants were students from a tertiary allied health profession; (b) the intervention was explicit cultural competence training in any setting; (c) the journal article was peer-reviewed; (d) the quantitative data were designed to demonstrate a change in skill. Studies were excluded if: (a) the participants were nursing, medical, dental or psychology counselling students, or if data could not be extracted from samples that included students from the excluded populations; (b) the training occurred through immersion in fieldwork/placement without reference to cultural competence; (c) the study only reported a survey of courses or evaluation of an assessment tool; or d) the publication was in a language other than English.

The results of the search from each database were transferred into EndNote 7 (Clarivate Analytics), and duplicates were removed. The remaining studies were transferred into Covidence ${ }^{\odot}$ (Veritas Health Innovation) software. Each entry was screened by two reviewers. Conflicts between reviewers were managed in Covidence and resolved through consensus and a moderator was not required. Given the low number of included studies, reference list hand-searching of previously published related systematic reviews and all included studies was conducted to ensure all relevant research were identified.

\section{Data Coding}

A data coding worksheet in $\mathrm{Excel}^{-}$(Microsoft) was created and piloted on three studies and refined. All the included studies were coded by two reviewers for the following information: study characteristics (including aim/objectives; research design; definition of cultural competence), participant characteristics (including inclusion/exclusion characteristics; recruitment; age; gender; ethnicity; education level; program), intervention, and setting (including location of delivery; occurrence of clinical practice; content; format; 
time; and time frame of intervention; description of provider; theoretical basis), and outcomes (including quantitative outcome measures; data analysis; significance of results; costs). Data coding was compared for accuracy and disagreements were resolved through consensus between the reviewers and a moderator was not required. We contacted eight authors for further information. Three authors responded: two were not able to give any further information; and one provided additional information. ${ }^{9}$

\section{Data Analysis}

The included studies were each critically appraised by two reviewers using the Mixed Methods Appraisal Tool (MMAT) as it can adapt to different methodologies and has a good intra-class correlation of $0.72-0.94 .^{10}$ Appraisals were compared for accuracy and disagreements were resolved through consensus between the reviewers. A third reviewer moderated one discrepancy to determine the outcome.

When available, descriptive (means, standard deviations) and inferential statistical information ( $p$ values) were extracted from the quantitative findings and collated as principal summary measures for analysis. When adequate data were reported, standardized mean differences (SMDs) were calculated. Standardized mean differences are a measure of effect size and were calculated as the mean difference in cultural competence outcome between pre-training and post-training time points, and between trained and non-trained (control) results, divided by the pooled standard deviation, with adjustment for small sample sizes (Hedges' $g$ ). ${ }^{11}$ Effect sizes were interpreted as follows: $0.2=$ small; $0.5=$ medium; and $0.8=$ large..$^{12}$ Standardized Mean Differences are presented as Forest Plots as a visual aid to interpretation of the findings. Substantial study heterogeneity prevented pooling and meta-analysis of quantitative data, therefore, a best-evidence synthesis was conducted. ${ }^{13}$

\section{RESULTS}

Fifteen studies met all eligibility criteria and were included in the final review (Figure 1).

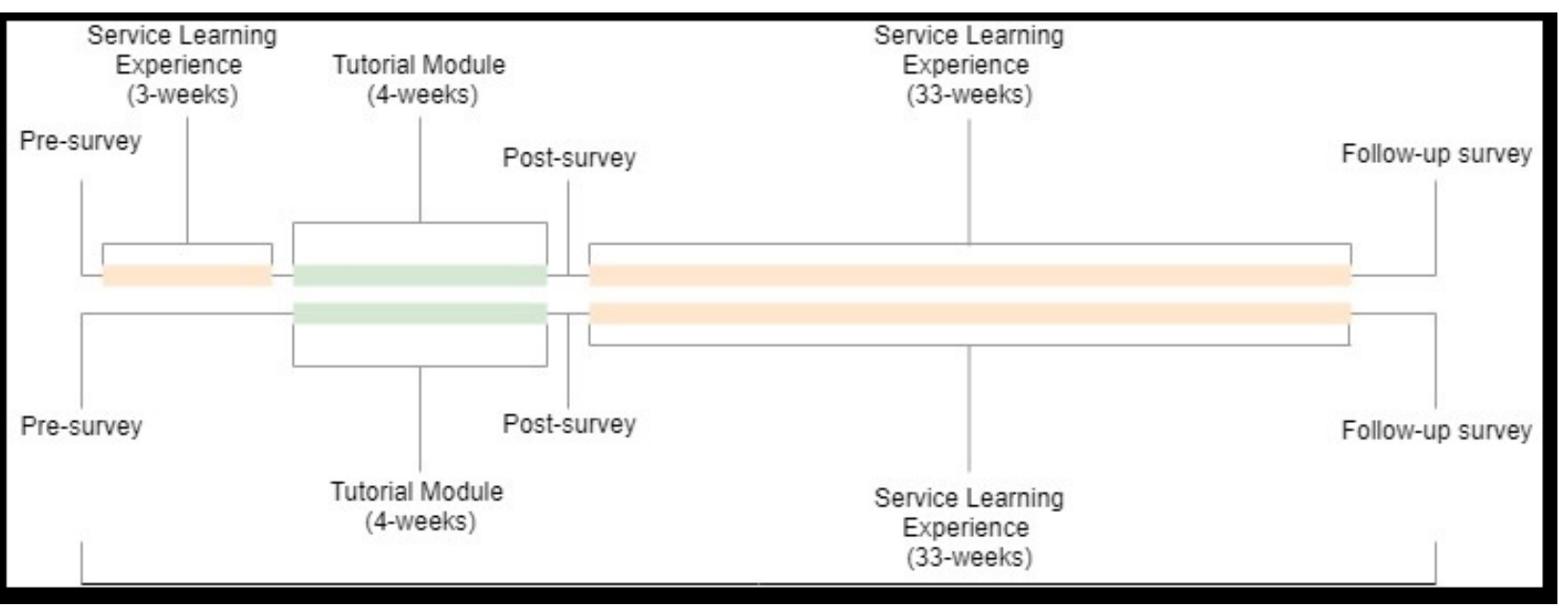

Figure 1. Timing of Assessments

\section{Characteristics of the Intervention Studies}

A total of 1284 allied health students from 15 studies were identified. The participant characteristics are presented in Table 1. In all but one of the included studies, students were attending universities in the USA. ${ }^{14}$ Most of the students were participating in pharmacy studies ( $n=9$ studies plus one multi-disciplinary study which also included physical therapy students) with three studies on physical therapy and two studies on occupational therapy students. Where data was reported, participants were predominately in the 20-25 years category; predominately female students (3:1); and Caucasian. 
Table 1. Participant Characteristics in the Included Studies

\begin{tabular}{|c|c|c|c|c|c|}
\hline & $\begin{array}{l}\text { Sample } \\
\text { (n) }\end{array}$ & Description & $\begin{array}{l}\text { Age } \\
\text { (years) }\end{array}$ & $\begin{array}{l}\text { Gender } \\
(\%)\end{array}$ & $\begin{array}{l}\text { Ethnicity } \\
(\%)\end{array}$ \\
\hline $\begin{array}{l}\text { Assemi et } \\
\text { al. } 2006\end{array}$ & 175 & $\begin{array}{l}\text { Pharmacy students; } \\
\text { 1st year } 45.2 \\
\text { 2nd year } 35.4 \\
\text { 3rd year } 18.3 \\
\text { 4th year } 1.1\end{array}$ & NR & $\begin{array}{l}\text { Female } 88 \\
\text { Male } 12\end{array}$ & NR \\
\hline $\begin{array}{l}\text { Poirier et } \\
\text { al. } 2009\end{array}$ & 77 & Third year Pharmacy students & NR & NR & NR \\
\hline $\begin{array}{l}\text { Musolino } \\
\text { et al. } 2010\end{array}$ & 1974 & $\begin{array}{l}\text { Students enrolled in Medicine, } \\
\text { Nursing, Physical Therapy, } \\
\text { Pharmacy and others } \\
\text { Control group third year Physical } \\
\text { Therapy and Medicine students }\end{array}$ & NR & $\begin{array}{l}\text { Female } 63 \\
\text { Male } 34\end{array}$ & $\begin{array}{l}\text { Caucasian } 87 \\
\text { Asian } 5.8 \\
\text { Hispanic/Latino } 3 \\
\text { African American } 0.9 \\
\text { Native American } 0.6 \\
\text { Other } 2.6\end{array}$ \\
\hline $\begin{array}{l}\text { Muzumdar } \\
\text { et al. } 2010\end{array}$ & 85 & Second year Pharmacy students & NR & $\begin{array}{l}\text { Female } 65.9 \\
\text { Male } 34.1\end{array}$ & $\begin{array}{l}\text { Caucasian } 85.9 \\
\text { Asian } 12.9 \\
\text { Others } 1.2\end{array}$ \\
\hline $\begin{array}{l}\text { Durand et } \\
\text { al. } 2012\end{array}$ & 12 & Second year Pharmacy students & NR & NR & NR \\
\hline $\begin{array}{l}\text { Haack \& } \\
\text { Phillips } \\
2012\end{array}$ & 135 & $\begin{array}{l}\text { First year Doctor of Pharmacy } \\
\text { students }\end{array}$ & NR & NR & Caucasian 86.7 \\
\hline $\begin{array}{l}\text { Sales et } \\
\text { al. } 2013\end{array}$ & 84 & Second-year Pharmacy students & $20-2593 \%$ & $\begin{array}{l}\text { Female } 42.9 \\
\text { Male } 57.1\end{array}$ & Caucasian 81 \\
\hline $\begin{array}{l}\text { Gat \& } \\
\text { Ratzon } \\
2014\end{array}$ & 50 & $\begin{array}{l}\text { Bachelor of Occupational } \\
\text { Therapy students who had } \\
\text { completed their first, second, or } \\
\text { third fieldwork placements }\end{array}$ & $\begin{array}{l}\text { Mean } 23.5 \\
(S D \text { 3.3). }\end{array}$ & $\begin{array}{l}\text { Female }(n=24) \\
\text { Males NR } \\
\text { Controls NR }\end{array}$ & NR \\
\hline $\begin{array}{l}\text { Hayward } \\
\& \text { Li } 2014\end{array}$ & 14 & $\begin{array}{l}\text { Fifth year Doctor of Physical } \\
\text { Therapy students }\end{array}$ & Average 23 & $\begin{array}{l}\text { Female } 85.7 \\
\text { Male } 14.3\end{array}$ & Ethnicity/minority 28.5 \\
\hline $\begin{array}{l}\text { Cailor \& } \\
\text { Chen } \\
2015\end{array}$ & 53 & First year Pharmacy students & $\begin{array}{l}20-2578 \% \\
26-309.5 \% \\
31-353.8 \% \\
36-401.9 \% \\
46-501.9 \% \\
\text { Missing } \\
5.7 \% \\
\text { Median } 21.5\end{array}$ & $\begin{array}{l}\text { Female } 60.4 \\
\text { Male } 39.6\end{array}$ & $\begin{array}{l}\text { Caucasian } 85 \\
\text { African/American } 5.7 \\
\text { Asian or Pacific Islander } \\
5.7 \\
\text { Other } 3.8\end{array}$ \\
\hline $\begin{array}{l}\text { Crawford } \\
\text { et al. } 2016\end{array}$ & 537 & $\begin{array}{l}\text { All years of Doctor of Pharmacy } \\
\text { students }\end{array}$ & $\begin{array}{l}21-2548 \% \\
26-3042 \% \\
31-4910 \%\end{array}$ & $\begin{array}{l}\text { Female } 64 \\
\text { Male } 36\end{array}$ & $\begin{array}{l}\text { Caucasian } 52 \\
\text { Asian } 29 \\
\text { Other } 19\end{array}$ \\
\hline $\begin{array}{l}\text { Denton et } \\
\text { al. } 2016\end{array}$ & 52 & $\begin{array}{l}\text { First year Doctor of Physical } \\
\text { Therapy students }\end{array}$ & $\begin{array}{l}\text { Mean } 23.5 \\
(S D 2.4)\end{array}$ & $\begin{array}{l}\text { Female } 52 \\
\text { Male } 48\end{array}$ & $\begin{array}{l}\text { Caucasian } 54 \\
\text { Hispanic/Latino } 31 \\
\text { Asian } 10 \\
\text { African American } 2 \\
\text { Other } 4\end{array}$ \\
\hline $\begin{array}{l}\text { Paparella- } \\
\text { Pitzel et } \\
\text { al. } 2016\end{array}$ & 37 & $\begin{array}{l}\text { Second year Doctor of Physical } \\
\text { Therapy students }\end{array}$ & NR & $\begin{array}{l}\text { Female } 68 \\
\text { Male } 48\end{array}$ & $\begin{array}{l}\text { Caucasian } 69 \\
\text { African America } 11 \\
\text { Asian } 8.5 \\
\text { Hispanic } 8.5 \\
\text { Other } 1\end{array}$ \\
\hline $\begin{array}{l}\text { Arif et al. } \\
2017\end{array}$ & 159 & Third year Pharmacy students & Mean 25.6 & $\begin{array}{l}\text { Female } 66 \\
\text { Male } 34\end{array}$ & NR \\
\hline
\end{tabular}




\begin{tabular}{|c|c|c|c|c|c|}
\hline $\begin{array}{l}\text { Keane \& } \\
\text { Provident } \\
2017\end{array}$ & 12 & $\begin{array}{l}\text { Masters of Occupational Therapy } \\
\text { and Physical Therapy students }\end{array}$ & Mean 24.9 & $\begin{array}{l}\text { Female } 100 \\
\text { Male } 0\end{array}$ & $\begin{array}{l}\text { Caucasian } 75 \\
\text { Asian } 17 \\
\text { Hispanic } 8\end{array}$ \\
\hline
\end{tabular}

NR - Not Reported

The characteristics of the studies are summarised in Table 2. Ten of the included studies were pre-post study design, two were mixed method pre-post studies, one cross-sectional, one quasi experimental and one non-randomised intervention study. All included studies were published since 2006. Five studies developed curricula for their intervention training based on the model by Campinha-Bacote and three studies used a literature review as the foundation for the intervention course design. The work of Purnell, the Well's Cultural Development Model and the SOLER and LEARN communication models were each used once. Four studies, however, did not describe their theoretical model.

No study described the instructor's skills or quality of delivery. Cultural competence training was delivered exclusively in the classroom in eight studies, whereas five studies described classroom and fieldwork interventions and one study was based solely on fieldwork. Classroom hours ranged from 10 minutes to 30 hours and fieldwork ranged from 40 hours to 280 hours. Learning activities used in the interventions included readings; classroom discussion; lectures; role plays; videos; online discussion; group work; and reflective writing; laboratory practice; and field experience.

Given that the review question was based on culture, there is a risk of bias across the studies as 14 of the included studies were conducted in the USA, with only one study conducted in another country (Israel). ${ }^{14}$ No author appeared on more than one study, although there may be a publication bias as over a third $(n=6)$ of the studies were published in the American Journal of Pharmaceutical Education. All the other studies were in different journals. The risk for bias within studies based on issues of methodological rigour is summarized in Table 3. The rigour of five studies was threatened because the participants volunteered to enrol in elective courses and this initial level of interest may have the potential to introduce a selection bias. ${ }^{9,15,18,19,20} \mathrm{~A}$ pre-post study design was most common, and no study utilized a long-term follow-up period in their design. This meant that this review is unable to determine whether any change in cultural competence skill is transitory or enduring. Although the IPACC has good reliability and validity, a number of studies failed to report this. All the studies used a self-report instrument, so no objective data were obtained on skill development. This means the review is unable to comment on the degree or quality of actual cultural competence skill change. 
Table 2. Summary of Studies

\begin{tabular}{|c|c|c|c|c|c|}
\hline Reference & $\begin{array}{l}\text { Study } \\
\text { sample }\end{array}$ & Intervention curricula content & $\begin{array}{l}\text { Intervention teaching } \\
\text { strategies }\end{array}$ & $\begin{array}{l}\text { Intervention } \\
\text { duration }\end{array}$ & Individual skill outcome variable (mean \pm SD significance) \\
\hline $\begin{array}{l}\text { Assemi, } \\
\text { Cullander \& } \\
\text { Hudmon } \\
2006^{\mathrm{a} 1}\end{array}$ & $\begin{array}{l}175 \\
\text { Pharmacy } \\
\text { students }\end{array}$ & $\begin{array}{l}\text { Based on accreditation } \\
\text { requirements: } \\
\text { Culturally competent attitudes, } \\
\text { knowledge, and skills }\end{array}$ & $\begin{array}{l}\text { Classroom } \\
\text { Discussions, lectures, role } \\
\text { plays, videos }\end{array}$ & 8 hours $^{\mathrm{b} 1}$ & $\begin{array}{l}\text { Elicit a patient's perspective of illness during a patient encounter; pre: } \\
2.50 \pm 0.76 \text {, post: } 3.84 \pm 0.66, p<0.001 \\
\text { Elicit a patient's perspective of healing and medication therapy during a } \\
\text { patient encounter; pre: } 2.49 \pm 0.75 \text {, post: } 3.83 \pm 0.67, p<0.001 \\
\text { Effectively monitor the therapy of a patient from a background different } \\
\text { from your own; pre: } 2.76 \pm 0.83 \text {, post: } 3.87 \pm 0.64, p<0.001 \\
\text { Effectively counsel a patient from a background different from your own } \\
\text { on their meds or supplements; pre: } 2.76 \pm 0.84 \text {, post: } 3.87 \pm 0.66, p< \\
0.001 \\
\text { Effectively utilize an unskilled interpreter to interview or counsel a patient; } \\
\text { pre: } 2.40 \pm 0.81 \text {, post: } 4.16 \pm 0.63, p<0.001 \\
\text { Effectively utilize a skilled interpreter to interview or counsel a patient; pre: } \\
2.82 \pm 0.92 \text {, post: } 4.33 \pm 0.60, p<0.001\end{array}$ \\
\hline $\begin{array}{l}\text { Poirier et al. } \\
2009^{a} 1\end{array}$ & $\begin{array}{l}77 \\
\text { Pharmacy } \\
\text { students }\end{array}$ & $\begin{array}{l}\text { Based on work of Campinha- } \\
\text { Bacote: } \\
\text { Culturally competent self- } \\
\text { awareness, knowledge, and } \\
\text { skills, including health } \\
\text { promotion strategies for } \\
\text { providing culturally responsive } \\
\text { care }\end{array}$ & $\begin{array}{l}\text { Classroom team-based } \\
\text { learning approach } \\
\text { preassigned readings, group } \\
\text { work }\end{array}$ & $\begin{array}{l}\text { Spread over } 3 \\
\text { class } \\
\text { sessions }^{\text {b2 }}\end{array}$ & Cultural skill; pre: $11.8 \pm 1.7$, post: $15.7 \pm 2.0, p<0.001$ \\
\hline $\begin{array}{l}\text { Musolino et } \\
\text { al. } 2010^{\mathrm{a} 1}\end{array}$ & $\begin{array}{l}82 \text { Physical } \\
\text { Therapy } \\
\text { and } 102 \\
\text { Pharmacy } \\
\text { students }\end{array}$ & $\begin{array}{l}\text { Based on un- named nationally } \\
\text { recognised expert in CC. } \\
\text { Accountability and mutual } \\
\text { respect; attitudes, beliefs and } \\
\text { expectations: disparity of care } \\
\text { and relationships; systems } \\
\text { diversity: solutions to cultural } \\
\text { clashes; cross-cultural } \\
\text { communication }\end{array}$ & Classroom & $\begin{array}{l}8 \text { hours of } \\
\text { modules }\end{array}$ & $\begin{array}{l}\text { Physical therapy: Cultural skill; pre: } 12.3 \text {, post: } 13.9^{*} \nabla \\
\text { Pharmacy: Cultural skill; pre: } 12.3, \text { post: } 14.2^{* \nabla}\end{array}$ \\
\hline
\end{tabular}

(c) The Internet Journal of Allied Health Sciences and Practice, 2022 


\begin{tabular}{|c|c|c|c|c|c|}
\hline $\begin{array}{l}\text { Muzumdar, } \\
\text { Holiday- } \\
\text { Goodman, } \\
\text { Black \& } \\
\text { Powers } \\
2010^{\text {a2 }}\end{array}$ & $\begin{array}{l}85 \\
\text { Pharmacy } \\
\text { students. }\end{array}$ & $\begin{array}{l}\text { Based on a literature review: } \\
\text { Cultural awareness }\end{array}$ & $\begin{array}{l}\text { Classroom } \\
\text { case studies, group work, } \\
\text { lectures, readings, reflective } \\
\text { writings, videos, written } \\
\text { assignment } \\
\text { Fieldwork } \\
\text { Visiting diverse settings, } \\
\text { patients interviews, attending } \\
\text { a diverse group }\end{array}$ & Not reported ${ }^{\mathrm{b} 1}$ & $\begin{array}{l}\text { Kleinman Question; pre : } 0.7 \pm 1.1 \text {, post } 2.8 \pm 1.0, p<0.01 \\
\text { Patient-centered clinical model; pre: } 1.1 \pm 1.0, \text { post: } 3.1 \pm 0.9, p<0.01 \\
\text { LEARN model; pre: } 0.6 \pm 1.0 \text {, post: } 3.0 \pm 1.0, p<0.01 \\
\text { RESPECT model; pre: } 0.6 \pm 0.9 \text {, post: } 3.0 \pm 1.0, p<0.01 \\
\text { ADHERE model; pre: } 0.9 \pm 0.8 \text {, post: } 2.7 \pm 1.0, p<0.01 \\
\text { Teachback method pre: } 2.9 \pm 1.2 \text {, post: } 4.0 \pm 0.9, p<0.01\end{array}$ \\
\hline $\begin{array}{l}\text { Durand, } \\
\text { Abel, Silva } \\
\text { \& Desilets } \\
2012^{a 1}\end{array}$ & $\begin{array}{l}12 \\
\text { Pharmacy } \\
\text { students }\end{array}$ & $\begin{array}{l}\text { Basis not stated: } \\
\text { Culturally competent attitudes, } \\
\text { knowledge, and skills including } \\
\text { how to overcome barriers to } \\
\text { care, and create patient- } \\
\text { suitable health screening } \\
\text { literature }\end{array}$ & $\begin{array}{l}\text { Classroom } \\
\text { Interactive sessions with } \\
\text { community, lectures, online } \\
\text { discussion, written } \\
\text { assignment }\end{array}$ & $\begin{array}{l}3 \text { hours } / 10 \\
\text { weeks }^{\text {b3 }}\end{array}$ & $\begin{array}{l}\text { Cultural skills; pre:7, post: }{ }^{*} \text {, } \\
p=0.1\end{array}$ \\
\hline $\begin{array}{l}\text { Haack \& } \\
\text { Phillips } \\
2012^{a 3}\end{array}$ & $\begin{array}{l}135 \\
\text { Pharmacy } \\
\text { students }\end{array}$ & $\begin{array}{l}\text { Based on a literature review: } \\
\text { Culturally competent attitudes, } \\
\text { knowledge, and skills including } \\
\text { communicating with culturally } \\
\text { diverse patient populations, } \\
\text { self-reflection, working with } \\
\text { interpreters and eliciting } \\
\text { patients' health beliefs }\end{array}$ & $\begin{array}{l}\text { Classroom } \\
\text { Laboratory work, lectures } \\
\text { Fieldwork (Experiential } \\
\text { learning opportunities) } \\
\text { Interactions with patients }\end{array}$ & $\begin{array}{l}4 \text { hours of } \\
\text { laboratory } \\
2 \text { hours of } \\
\text { lecture } \\
40-42 \text { hours } \\
\text { patient } \\
\text { interaction }{ }^{\text {b2 }}\end{array}$ & Cultural skills; control: $12.9 \pm 2.0$, training: $13.8 \pm 2.6, p=0.021$ \\
\hline $\begin{array}{l}\text { Sales, } \\
\text { Jonkman, } \\
\text { Connor \& } \\
\text { Hall 2013 }\end{array}$ & $\begin{array}{l}84 \\
\text { Pharmacy } \\
\text { students }\end{array}$ & $\begin{array}{l}\text { Basis not stated: } \\
\text { Culturally competent attitudes, } \\
\text { knowledge, and skills including } \\
\text { designing a monitoring plan for } \\
\text { patients }\end{array}$ & $\begin{array}{l}\text { Group } 1 \\
\text { Lecture including } 2 \text { case } \\
\text { scenarios } \\
\text { Group } 2 \\
\text { Lecture, written assignment } \\
\text { Group } 3 \\
\text { Lecture, interview a } \\
\text { simulated patient }\end{array}$ & $\begin{array}{l}\text { Group 1 } \\
50 \text { minutes } \\
\text { lecture } \\
\text { Group } 2 \\
10 \text { minutes } \\
\text { lecture + } 40 \\
\text { minutes written } \\
\text { group work } \\
\text { Group 3 } \\
10 \text { minutes } \\
\text { lecture + 40 } \\
\text { minutes } 2 \\
\text { simulated } \\
\text { patient } \\
\text { encounters }\end{array}$ & $\begin{array}{l}\text { Modifying one's interview when encountering diverse populations; pre: } \\
2.2 \text {, post: } 2.7^{*}, p<0.01\end{array}$ \\
\hline
\end{tabular}




\begin{tabular}{|c|c|c|c|c|c|}
\hline $\begin{array}{l}\text { Gat \& } \\
\text { Ratzon } \\
2014 \text { a5 }\end{array}$ & $\begin{array}{l}50 \\
\text { Occupation } \\
\text { al Therapy } \\
\text { students }\end{array}$ & $\begin{array}{l}\text { Basis not stated: } \\
\text { Fieldwork only }\end{array}$ & Fieldwork & $\begin{array}{l}1^{\text {st }} \text { years } \\
280 \text { hours } \\
2^{\text {nd }} \text { years } \\
315 \text { hours } \\
3^{\text {rd }} \text { years } \\
390 \text { hours }^{\text {b1 }}\end{array}$ & Cultural competence; control: $3.70 \pm 1.22$, training: $4.30 \pm 1.03, p=0.023$ \\
\hline $\begin{array}{l}\text { Hayward \& } \\
\text { Li 2014 }\end{array}$ & $\begin{array}{l}14 \text { Physical } \\
\text { Therapy } \\
\text { students }\end{array}$ & $\begin{array}{l}\text { Based on the work of Purnell } \\
\text { and Campinha -Bacote. } \\
\text { Developed own blended model } \\
\text { with an emphasis on advocacy }\end{array}$ & $\begin{array}{l}\text { Classroom } \\
\text { Discussion, reading, self- } \\
\text { awareness activity } \\
\text { Fieldwork } \\
\text { Cultural encounter in } \\
\text { Ecuador }\end{array}$ & $\begin{array}{l}24 \text { class room } \\
\text { hours } \\
9 \text { day fieldwork } \\
\text { tripb3 }\end{array}$ & Cultural skill; pre: $7.5 \pm 0.94$, post: $9.0 \pm 1.4, p=0.002$ \\
\hline $\begin{array}{l}\text { Cailor \& } \\
\text { Chen } \\
2015^{\mathrm{a} 1}\end{array}$ & $\begin{array}{l}53 \\
\text { Pharmacy } \\
\text { students }\end{array}$ & $\begin{array}{l}\text { Based on a literature review } \\
\text { including the work of } \\
\text { Campinha-Bacote. } \\
\text { Cultural competent knowledge, } \\
\text { and skills including health } \\
\text { literacy }\end{array}$ & $\begin{array}{l}\text { Classroom } \\
\text { Laboratory, lectures }\end{array}$ & $\begin{array}{l}3 \text { hours self- } \\
\text { care } \\
1 \text { hour lecture, } \\
4.5 \text { hours } \\
\text { laboratoryb3 }\end{array}$ & Cultural skills; pre: $7.4 \pm 1.71$, post: $9.25 \pm 1.54, p<0.001^{\#}$ \\
\hline $\begin{array}{l}\text { Crawford et } \\
\text { al. } 2016^{\text {a1 }}\end{array}$ & $\begin{array}{l}150 \\
\text { Pharmacy } \\
\text { students }\end{array}$ & $\begin{array}{l}\text { Based on Wells' Cultural } \\
\text { Development Model. } \\
\text { Health literacy, health beliefs, } \\
\text { patient explanatory models, } \\
\text { social determinants, health } \\
\text { disparities }\end{array}$ & $\begin{array}{l}\text { Classroom } \\
\text { Practical experience }\end{array}$ & 6 hours $^{b 4}$ & $\begin{array}{l}\text { Cultural skill; pre: } 43.7 \pm 20.9 \text {, post: } 56.1 \pm 19.4, p<0.001 \\
\text { Comfort - clinical encounters; pre: } 52.7(21.5) \text {, post: } 59.0(18.9), p=0.012\end{array}$ \\
\hline $\begin{array}{l}\text { Denton et } \\
\text { al. } 2016^{\text {a1 }}\end{array}$ & $\begin{array}{l}52 \text { Physical } \\
\text { Therapy } \\
\text { students }\end{array}$ & $\begin{array}{l}\text { Based on the work of } \\
\text { Campinha-Bacote } \\
\text { Health disparities; social } \\
\text { determinants; Kleinman's } \\
\text { questions; use of interpreter }\end{array}$ & Classroom & $\begin{array}{l}12 \text { classroom } \\
\text { hours } \\
+4 \text { hours over } \\
2 \text { visits }{ }^{\text {b3 }}\end{array}$ & Cultural skill; pre: 7.29 , post: $9.5 p<0.001^{\#^{*}}$ \\
\hline $\begin{array}{l}\text { Paparella- } \\
\text { Pitzel, } \\
\text { Eubanks \& } \\
\text { Kaplan } \\
2016^{\mathrm{a} 1}\end{array}$ & $\begin{array}{l}42 \text { Physical } \\
\text { Therapy } \\
\text { students }\end{array}$ & $\begin{array}{l}\text { Based on the work of } \\
\text { Campinha-Bacote and concept } \\
\text { of cultural humility. } \\
\text { Cultural competence, } \\
\text { constructs of dialogue, patient } \\
\text { empowerment, analysis of }\end{array}$ & Classroom & $\begin{array}{l}4 \text { hours of } \\
\text { lectures }^{\mathrm{b} 2}\end{array}$ & Cultural skill; pre: $12.6 \pm 2.5$, post: $14.6 \pm 2.2, p<0.001^{\wedge}$ \\
\hline
\end{tabular}

(C) The Internet Journal of Allied Health Sciences and Practice, 2022 
environment, available

resources, development of self-

awareness

+ volunteers did an additional 2

hours on ethnocentrism

transcultural assessment

models, cultural brokering,

racism, prejudice, ethnicity,

awareness, techniques for

culturally aware interviews

\begin{tabular}{|c|c|c|c|c|c|}
\hline $\begin{array}{l}\text { Arif et al. } \\
2017^{a 1}\end{array}$ & $\begin{array}{l}159 \\
\text { Pharmacy } \\
\text { students }\end{array}$ & $\begin{array}{l}\text { Based on communication } \\
\text { models SOLER and LEARN. } \\
\text { Common patient beliefs; } \\
\text { barriers to cross-cultural } \\
\text { communication; active listening; } \\
\text { communication tools to } \\
\text { overcome barriers }\end{array}$ & Classroom & $\begin{array}{l}2 \text { hours } \\
\text { lectures in first } \\
\text { year } \\
1 \text { hour lecture } \\
+2 \text { hour } \\
\text { workshop in } \\
\text { third yearb1 }\end{array}$ & Cross cultural communication skill; pre: 55.5 , post: $56.6, p<0.05^{\star}$ \\
\hline $\begin{array}{l}\text { Keane \& } \\
\text { Provident } \\
2017^{a 1}\end{array}$ & $\begin{array}{l}6 \\
\text { Occupation } \\
\text { al Therapy } \\
\text { and } 6 \\
\text { Physical } \\
\text { Therapy }\end{array}$ & $\begin{array}{l}\text { Based on the work of } \\
\text { Campinha-Bacote } \\
\text { Cultural awareness and } \\
\text { knowledge +language and } \\
\text { treatment skills }\end{array}$ & $\begin{array}{l}\text { Classroom } \\
\text { Fieldwork }\end{array}$ & $\begin{array}{l}\text { classroom/ } \\
\text { online hours } \\
\text { not reported } \\
9 \text { day } \\
\text { immersion }\end{array}$ & Cultural skill; pre: 6.7 , post: $8.7 p<0.001^{*}$ \\
\hline
\end{tabular}




\section{Effects of the Interventions}

The effect of cultural competence training was assessed using either a self-developed questionnaire or survey instrument, or a modified version of the Clinical Cultural Competency Questionnaire (mCCCQ) or a version of the Inventory for Assessing the Process of Cultural Competence (IAPCC), with five studies using the student version and the other four using the revised version. The IAPCC question deemed to reflect actual or perceived skill change (as per the inclusion criteria) was 'Cultural Skill'. The Cultural Skill subscale has demonstrated adequate internal consistency (Cronbach alpha 0.72 retest cohort), and as such was deemed appropriate to use as an outcome. ${ }^{21}$ The $\mathrm{mCCCQ}$ used by Crawford and colleagues includes items on skills in greeting patients in culturally sensitive manner, eliciting patient perspectives on health and illness, providing dosage form alternatives, recommending treatment alternatives associated with racial/ethnic differences in drug metabolism, assessing patients' health literacy, working with medical interpreters, and dealing with adherence problems for cross-cultural populations. ${ }^{16}$ Assemi and colleagues used a 12-item survey instrument measuring student's self-reported perceptions of their cultural competence awareness, knowledge and skills. ${ }^{22}$ Data were coded for six of the 12 survey items that reflected students' actual or perceived skill change (Table 3). Muzumdar and colleagues used a survey with 32 items, but only six items were deemed to reflect perceived or actual change in skills. ${ }^{23}$ Sales and colleagues used a 12-item cultural assessment survey of which one item was deemed to reflect the research question. ${ }^{19}$ Gat and Ratzon administered a 29-item questionnaire to students who had participated in either a traditional fieldwork (control) or community fieldwork (training) placement and data from one cultural competence skill-related question was coded. ${ }^{14}$ 
Table 3. Critical Appraisal of Included Studies using the Mixed Methods Appraisal Tool10

\begin{tabular}{|c|c|c|c|c|c|c|c|}
\hline Study & & & Mixed Me & ods Appraisal Tc & Question & & \\
\hline & $\begin{array}{l}3.1^{1} \\
\text { Recruitment }\end{array}$ & \begin{tabular}{|l|}
3.2 \\
Measurement \\
tools
\end{tabular} & $\begin{array}{l}3.3 \text { Group } \\
\text { comparability }\end{array}$ & $\begin{array}{l}3.4 \text { Outcome } \\
\text { data }(>80 \%) \text { or } \\
\text { response rate } \\
(>60 \%)\end{array}$ & $\begin{array}{l}5.1^{2} \text { Research } \\
\text { design }\end{array}$ & $\begin{array}{l}5.2 \text { Design } \\
\text { justification }\end{array}$ & 5.3 Limitations \\
\hline Assemi, Cullander \& Hudmon, 2006 & Yes & Yes & Yes & Yes & & & \\
\hline Poirier et al., 2009 & Yes & Can't Tell & Yes & Yes & & & \\
\hline $\begin{array}{l}\text { Musolino, } 2010 \text { (quant data no SD not } \\
\text { meta-analysis) }\end{array}$ & Yes & Yes & Yes & No & & & \\
\hline $\begin{array}{l}\text { Muzumdar, Holiday-Goodman, Black \& } \\
\text { Powers, } 2010\end{array}$ & Yes & Yes & Yes & Yes & No & Yes & Can't Tell \\
\hline Durand, Abel, Silva \& Desilets, 2012 & Can't Tell & Can't Tell & Yes & Yes & & & \\
\hline Haack \& Phillips 2012 & Yes & Can't Tell & Can't Tell & Yes & & & \\
\hline Sales, Jonkman, Connor \& Hall, 2013 & Can't Tell & Can't Tell & Yes & Yes & & & \\
\hline Gat \& Ratzon, 2014 & Yes & Yes & Yes & No & & & \\
\hline Hayward \& Li, 2014 & No & Can't Tell & Yes & Yes & Yes & Yes & No \\
\hline Cailor \& Chen, 2015 & Yes & Can't Tell & Yes & Yes & & & \\
\hline Crawford et al., 2016 & Yes & Can't Tell & Yes & Yes & & & \\
\hline Denton et al., 2016 & Yes & Can't Tell & Yes & Yes & & & \\
\hline $\begin{array}{l}\text { Paparella-Pitzel, Eubanks \& Kaplan, } \\
2016\end{array}$ & No & Can't Tell & Yes & No & & & \\
\hline Arif et al., 2017 & Yes & No & Yes & Yes & & & \\
\hline Keane \& Provident, 2017 & No & Can't Tell & Yes & No & Yes & No & No \\
\hline
\end{tabular}

Note: Study design. 1 = Quantitative Non Randomized, 2 = Mixed Methods.

Section 2-Quantitative randomized controlled trials and section 4 Quantitative descriptive were not used in this table as there were no relevant studies. 
All 15 included studies provided quantitative data that reported a positive change in skill after cultural competence training (Table 2). Only one study reported a non-significant positive trend. ${ }^{18}$ Six studies did not provide standard deviation data, hence SMDs could not be calculated (Table 3). Forest Plots in Figure 2 and Figure 3 illustrate the SMD and 95\% confidence intervals for the individual cultural competence survey items that were deemed to reflect skill change. Figure 2 illustrates the data from the studies that used a comparative experimental design (control and intervention data) and Figure 3 illustrates the data from studies that used a pre-post design. Each line of data in the figures shows one outcome variable. The study's first author and a brief explanation of each outcome variable is provided on the left of both Forest plots. On the right of the Forest plots, the effect size of each outcome variable is represented by a block at the point estimate of the SMD with a horizontal line extending either side showing the $95 \%$ confidence interval of the SMD.

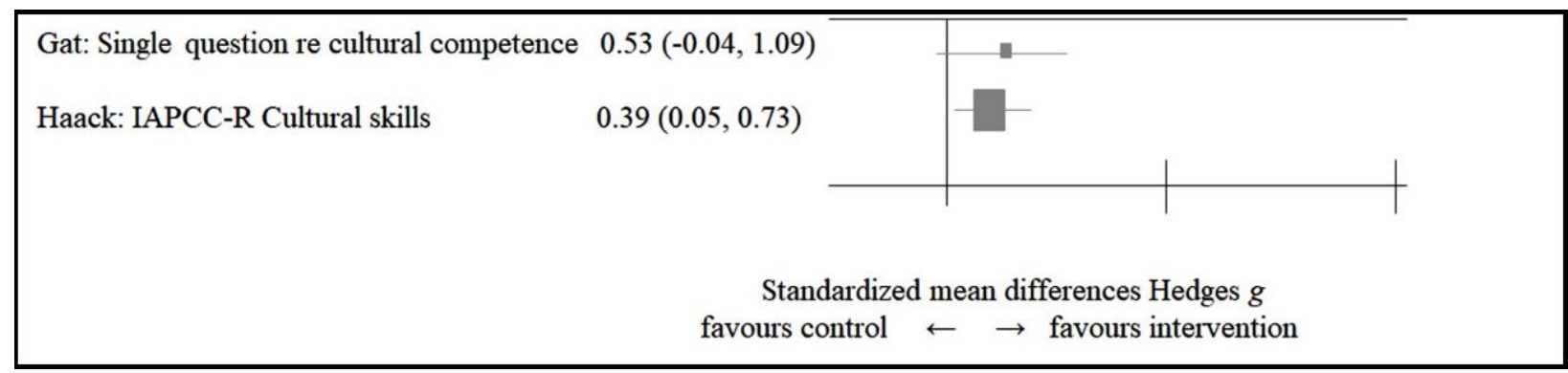

Figure 2. Data from the studies that used a comparative experimental design

One of the three studies that used a comparative experimental design (control and intervention data) did not provide adequate data to be included in Figure 2. ${ }^{19}$ The SMD for the Haack and Phillips study is $0.39(0.05-0.73)$ (Figure 2) which can be considered a small-medium effect size. ${ }^{3}$ The SMD for the Gat and Ratzon study is $0.53(-0.04-1.09$; (Figure 2) but this is a non-significant effect as the lower boundary of the $95 \% \mathrm{Cl}$ crosses zero. ${ }^{14}$

Five of the 12 studies that reported pre-post training data failed to report standard deviations, hence only seven studies could be analysed to report a SMD (Figure 3). The SMDs for studies that reported pre-post training scores without a comparator (Figure 3) range from $0.31-2.51$ suggesting a range from small to very large effect size. The SMD $95 \%$ confidence intervals for all included studies except Gat and Ratzon are statistically significant. ${ }^{14}$ 


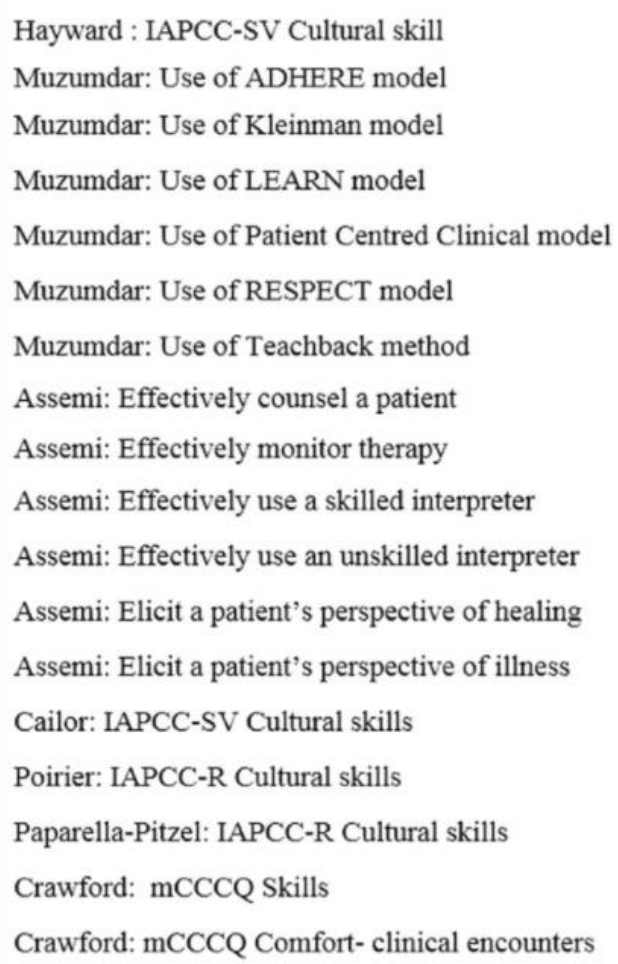

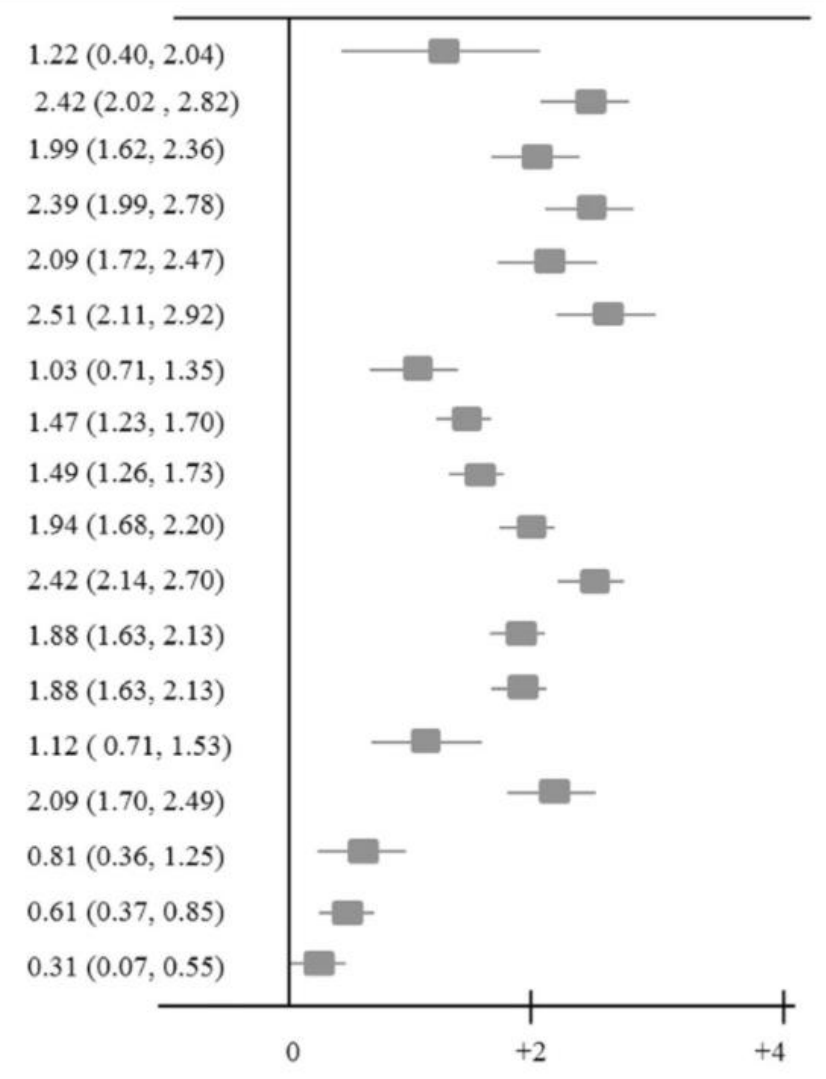

Standardized mean differences Heges $g$

favours pre measure $\leftarrow \quad \rightarrow$ favours post measure

Figure 3. Data from studies that used a pre-post design.

\section{DISCUSSION}

This review identified 15 studies that investigated the effectiveness of cultural competence training on developing culturally competent skills in allied health students. Fourteen of the 15 studies reported a significant positive impact, with allied health students reporting increases in cultural competence skills as a result of training. Effect sizes of the intervention training ranged from small to very large. Cultural competence training in the studies within this review was predominately conducted via traditional classroom teaching activities, although fieldwork is recognised was a valid learning setting in six studies. The assessment of cultural competence skills occurred through student self-assessment using versions of the IAPCC focussing on skills-related patient assessment tasks.

The review is limited to describing the perceived change in or use of skills due to the use of self-reported outcome measures by all the included studies. It is not possible to draw conclusions from the findings of this review regarding the translation of perceived skill to clinical practice or patient outcomes. Yet, there is nothing to say this would not be the case. Previous studies involving allied health professionals show a positive relationship between cultural competence training and improved patient satisfaction and patient outcomes.24,25 No studies in this review investigated objective measures of skill development (e.g. observation) or patient related outcomes.

Cultural competence in allied health curricula was conceptualized and operationalized by most of the included studies as a set of internal attributes within the student, such as their attitudes, knowledge and skills. ${ }^{18,22,23,26}$ Some studies extended this to a focus on the ability to practice effectively and respectfully. $9,17,23,27,28,29$ Others placed emphasis on the need to understand cultural competence as a process of continuous effort and improvement in working in different cultural contexts. ${ }^{16,20,19,20,28}$ We suggest future research should consider including the element of cultural distance, defined as the actual or perceived discrepancy between the culture of the student and the client as no studies in this review considered this. ${ }^{1}$ This may be particularly relevant to programs where the enrolment of students does not mirror the cultural diversity of the patient population. 
Four studies did not describe the conceptual model used to inform either the training or the outcome measure used. Nine studies used a version of the IAPCC as the self-report tool..$^{30}$ Filke and colleagues have suggested caution in the use of the IAPCC with different populations because there may be differences between predominately Caucasian and predominantly Hispanic populations..$^{21}$ All the studies used self-rating instruments which are susceptible to the bias of the participant about their own skill, which may have occurred in the context of this review by students wanting to achieve higher grades. The rigour of using self-report tools can be enhanced by gaining confirmation of results with inclusion of an external measure such as observational assessment by a peer, colleague or patient. The patient's voice should be an important part of the evaluation of a practitioner's cultural competence, but its omission is a common finding in the broader cultural competence literature. ${ }^{1}$ Further research should be conducted on the validity and reliability of fieldwork assessment tools and their ability to measure actual student cultural competence skills in practice and from the patient's perspective including quality of life or health outcomes. ${ }^{1}$

The review is biased towards studies that used a version of the IAPCC cultural competence skill domain. This domain defines cultural competence as "the ability to collect relevant cultural data regarding the client's presenting problem, as well as accurately performing a culturally-based, physical assessment in a culturally sensitive manner."31(p. 49) This definition and the subsequent questions in the IAPCC constrain the concept of cultural competence skill to patient assessment skills and don't include skills such as monitoring of therapy, counselling on medications or supplements and the ability to use a skilled or unskilled interpreter. ${ }^{22}$ The internal consistency of the IAPCC Cultural Skill subscale appears inconsistent between versions, with the Student Version demonstrating adequate internal consistency (Cronbach's alpha exceeding 0.70 ) but not so for the revised version. ${ }^{32}$ As a result, caution is advised when interpreting the findings of the studies.

\section{Limitations and Suggestions for Future Studies}

The review was limited to English-only studies, so some relevant studies may have been omitted. We also purposefully sought out peer-reviewed publications because of the intention to identify rigorous evidence based teaching strategies, but this means we may have missed some grey literature. The heterogeneity of included studies means we cannot make recommendations regarding the relative effectiveness of a single pedagogy, teaching strategy, or mode/context for such training. Meta-analysis was not possible because of the heterogeneity of study design and the number of studies reporting incomplete statistical data.

Cultural competence is not a new concept, but this review shows that research in effective training to achieve cultural competence is still developing. We suggest that some reorientation of the current research focus from investigating training effects on student knowledge or attitudes, towards studies on effective translation of this knowledge into culturally competent skills in allied health practice would help educators in designing their curricula. This is important because the enactment of implicit understandings into explicit behaviour is the determinant of the effect of allied health practitioners on patient experiences and outcomes. This will require study designs that progress from using measures of perceived skill change to tools that employ actual measures of skills in practice such as observation by educators or peers during a clinical placement, fieldwork or simulated health care environment. More research is needed into which year level training should be introduced, and what, if any, subsequent training is required. Research also needs to address the comparative effectiveness of classroom and fieldwork teaching strategies. Underpinning this are questions about pedagogy. For example, is a transformative learning approach more or less effective than an inquiry based approach? This review has uncovered the lack of detail reported about study participants and so little is known about the impact of life experience and the students' own cultural background on the effectiveness of cultural competence training.

\section{CONCLUSION}

This review of 15 studies provides preliminary evidence that cultural competence training provided for allied health students increases their cultural competence skills for clinical practice. To ensure the effective translation of skill development, tertiary institutions seeking to develop and evaluate cultural competence training programs should shift the focus from perceived skill development to actual clinical practice cultural competence skills that have evidence of producing improved patient outcomes. By enhancing cultural competence in allied health students, tertiary institutions are enabling students to use their understanding of health inequities to facilitate best practice working with people from diverse cultures within their own health care setting, as well as increase their capacity to work in other countries. 


\section{REFERENCES}

${ }^{*}$ References marked with an asterisk indicate studies included in the review:

1. Alizadeh S, Chavan M. Cultural competence dimensions and outcomes: A systematic review of the literature. Health Soc Care in the Community. 2016;24(6):e117-e130. http://doi:101111/hsc.12293

2. Brown B, Warren NS, Brehm B, et al. The design and evaluation of an interprofessional elective course with a cultural competence component. J Allied Health. 2008;37(1):e316-e337. http://doi:10.1016/S0749-3797(02)00657-8

3. $\quad{ }^{*}$ Haack S, Phillips C. Teaching cultural competency through a pharmacy skills and applications course series. Am J Pharm Ed. 2012;76(2):Article 27. http://doi:10.5688/ajpe76227

4. Anderson LM, Scrimshaw SC, Fullilove MT, Fielding JE, Normand J, Services TFoCP. Culturally competent health care systems: A systematic review. Am J Prev Med. 2003;24(3S):68-79. http://doi:10.1016/S0749-3797(02)00657-8

5. Beach MC, Price EG, Gary TL, et al. Cultural competence: A systematic review of health care provider educational interventions. Med Care. 2005;43(4):356-373. http://doi: 10.1097/01.mlr.0000156861.58905.96.

6. Chipps JA, Simpson B, Brysiewicz P. The effectiveness of cultural-competence training for health professionals in community-based rehabilitation: A systematic review of literature. Worldviews on Evid-Based Nurs. 2008;5:85-94. http://doi: 10.1111/j.1741-6787.2008.00117.x

7. Renzaho AMN, Romios P, Crock C, Sønderlund L. The effectiveness of cultural competence programs in ethnic minority patient-centred health care-a systematic review of the literature. Int J Qual Health Care. 2013;25(3):261-269. http://doi:10.1093/intqho/mzt006

8. Liberati A, Altman DG, Tetzlaf J, et al. The PRISMA statement for reporting systematic reviews and meta-analyses of studies that evaluate health care interventions: Explanation and elaboration. PLoS Med. 2009;6(7): e1000100. http://doi.10.1371/journal.pmed.1000100

9. $\quad$ *Paparella-Pitzel S, Eubanks R, Kaplan SL. Comparison of teaching strategies for cultural humility in Physical Therapy. J Allied Health. 2016;45(2):139-146. PMID: 27262472

10. Pace R, Pluye P, Bartlett G, et al. Testing the reliability and efficiency of the pilot Mixed Methods Appraisal Tool (MMAT) for systematic mixed studies review. Int J Nurs Stud. 2012;49:47-53. http://doi:10.1016/j.jnurstu.2011.07.002

11. Hedges LV. Distribution theory for Glass's estimator of effect size and related estimators. J Ed Stat. 1981;6(2):107128. http://doi:10.3102/10769986006002107

12. Cohen J. A power primer. Psychological Bulletin. 1992;112(1):155-159. http://doi:10.1037/0033-2909.112.1.155

13. Slavin RE. Best-evidence synthesis: An alternative to meta-analytic and traditional reviews. Education Researcher. 1986;15:5-11. http://doi:10.3102/0013189X015009005

14. *Gat S, Ratzon NZ. Comparison of occupational therapy students' perceived skills after traditional and nontraditional fieldwork Am J Occup Ther. 2014;68(2):e47-54. http://doi:10.5014/ajot.2014.007732

15. *Hayward LM, Li L. Promoting and assessing cultural competence, professional identity, and advocacy in Doctor of Physical Therapy (DPT) degree students within a community of practice. J Physical Ther Ed. 2014;28(1):23-36. http://doi: 10.1097/00001416-201410000-00005

16. ${ }^{*}$ Crawford SY, Awé C, Tawk RH, Pickard SA. A cross sectional and longitudinal study of pharmacy students perceptions of readiness to serve diverse populations. Am J Pharm Ed. 2016;80(4):Article 62. http://doi: 10.5688/ajpe80462

17. *Arif S, Cryder B, Mazan J, Quin ones -Boex A, Cyganska A. Using patient case video vignettes to improve students' understanding of cross-cultural communication. Am J Pharm Ed. 2017;81(3):56. http://doi: 10.5688/ajpe81356

18. *Durand C, Abel C, Silva M, Desilets A. An elective course in cultural competence. Currents in Pharmacy Teaching and Learning. 2012;4(2):102-110. http://doi:10.1016/j.cptl.2012.01.002

19. *Sales I, Jonkman L, Connor S, Hall D. A comparison of educational interventions to enhance cultural competency in pharmacy students. Am J Pharm Ed. 2013;77(4):Article 76. http://doi:10.5688/ajpe77476

20. ${ }^{*}$ Keane E, Provident I. Combining online education with international service learning to increase cultural competence. Internet Journal of Allied Health Science and Practice. 2017;15(3):Article 6.

21. Filke DS, Denton JM, Esparza S, Palombaro K. Calculations of minimal detectable change of construct subscales of a cultural competence instrument. J Physical Ther Ed. 2016;30(1):25-30. http://doi: 10.1097/00001416-20163001000004

22. *Assemi M, Cullander C, Hudmon KS. Psychometric analysis of a scale assessing self-efficacy for cultural competence in patient counselling. Ann Pharmacother. 2006;40(12):2130-2135. http://doi:10.1345/aph.1H248

23. *Muzumdar JM, Holiday-Goodman M, Black CM, Power M. Cultural competence knowledge and confidence after classroom activities. Am J Pharm Ed. 2010;74(8):150. http://doi: 10.5688/aj7408150 
24. Majumdar B, Browne G, Roberts J, Carpio B. Effects of cultural sensitivity training on health care provider attitudes and patient outcomes. J Nurs Scholarsh. 2004;36(2):161-166. http://doi: 10.1111/j.1547-5069.2004.04029.x.

25. Govere L, Govere EM. How effective is cultural competence training of healthcare providers on improving patient satisfaction of minority groups? A systematic review of literature. Worldviews on Evid-Based Nurs. 2016;13(6):402410. http://doi: 10.1111/wvn.12176.

26. "Musolino GM, Burkhalter ST, Crookston B, et al. Understanding and eliminating disparities in health care: development and assessment of cultural competence for interdisciplinary health professionals at the University of Utah: A 3-year investigation. J Physical Ther Ed. 2010;24(1):25-36. http://doi: 10.1097/00001416-201010000-00006

27. *Denton JM, Esparza S, Fike DS, Gonzalez J, Lundquist Denton M. Improvements in cultural competence through classroom and local cross-cultural service-learning activities. J Physical Ther Ed. 2016;30(2):6-13. http://doi.10.1080/10705420802255080

28. ${ }^{*}$ Cailor SM, Chen AMH. Immediate and longitudinal effects of incorporating health literacy and cultural competency into a yearlong pharmacy curriculum. Currents in Pharmacy Teaching and Learning. 2015;7(3):292-301. http://doi:10.1016/j.cptl.2014.12.005

29. Hawala-Druy S, Hill MH. Interdisciplinary: Cultural competency and culturally congruent education for millennials in health professions. Nurs Ed Today. 2012;32(7):772-778. http://doi:10.1016/j.nedt.2012.05.002

30. Campinha-Bacote, J. (1999). A model and instrument for addressing cultural competence in health care. Journal of Nursing education, 38(5), 203-207

31. Campinha-Bacote J. The process of cultural competence in the delivery of healthcare services: The journey continues. Cincinnati: Transcultural C.A.R.E. Associates; 2007.

32. Ho M-J, Lee K-L. Reliability and validity of three cultural competency measures. Med Ed. 2007;41(5):519. http://doi: 10.1111/j.1365-2929.2007.02747.x

33. *Poirier, T. I., Butler, L. M., Devraj, R., Gupchup, G. V., Santanello, C. \& Lynch, J. C. A. A cultural competency course for pharmacy students. Am J Pharm Ed. 2009;73(5), 81. http://doi: 10.5688/aj730581 


\section{APPENDIX: SCREENING FLOW DIAGRAM}

Adapted from Moher et al (2009)
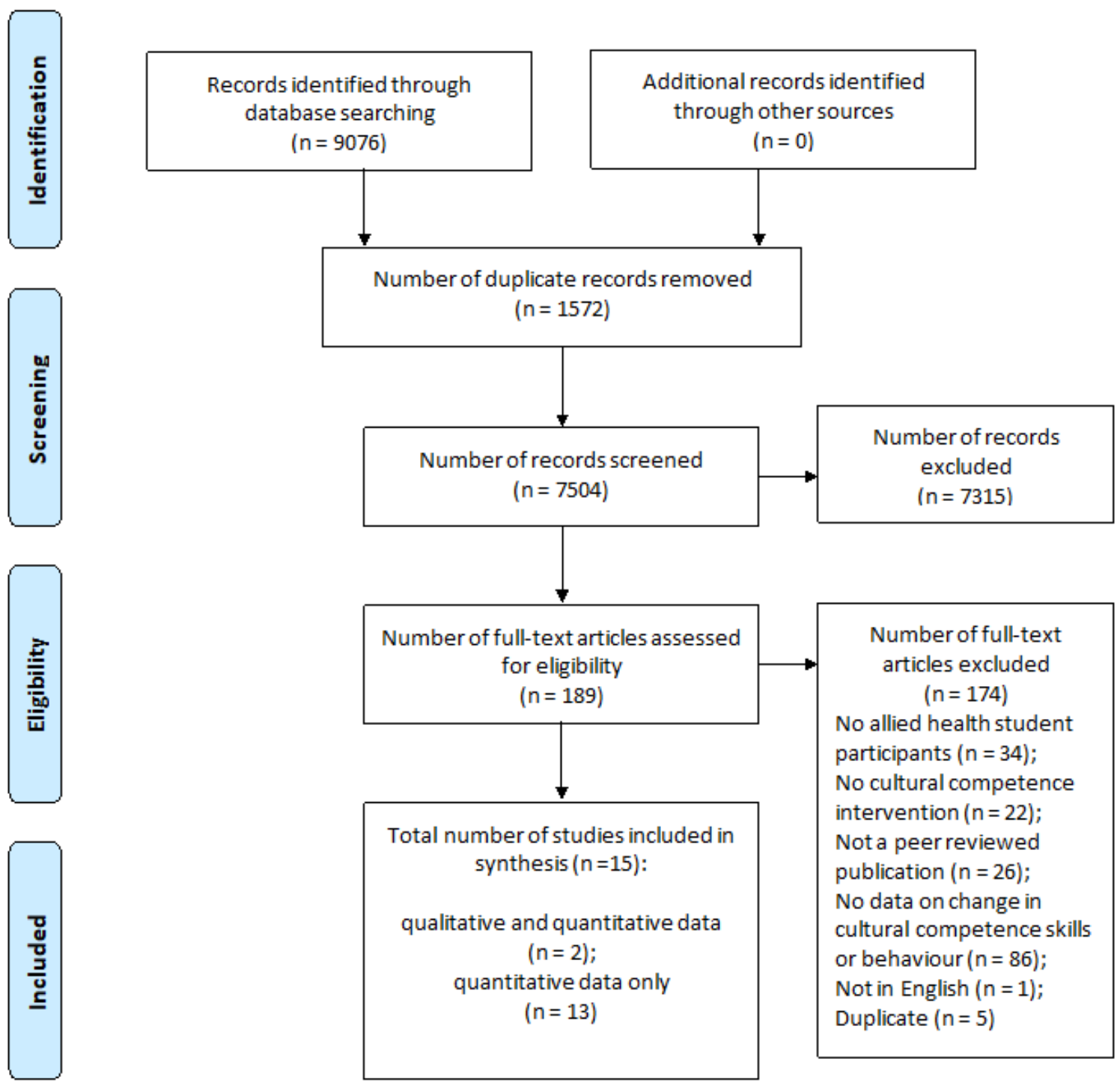